\title{
STRUCTURAL EVOLUTION OF THE TAYLOR VORTICES *
}

\author{
Tian MA ${ }^{1}$ AND SHOUHONG WANG $^{2}$
}

\begin{abstract}
We classify in this article the structure and its transitions/evolution of the Taylor vortices with perturbations in one of the following categories: a) the Hamiltonian vector fields, b) the divergencefree vector fields, and c). the solutions of the Navier-Stokes equations on the two-dimensional torus. This is part of a project oriented toward to developing a geometric theory of incompressible fluid flows in the physical spaces.
\end{abstract}

Résumé. Dans cet article, nous classons la structure et les transitions/évolutions des vortex de Taylor avec perturbations dans l'une des catégories suivantes : a) champs de vecteurs hamiltoniens, b) champs de vecteurs à divergence nulle, et c) solutions des équations de Navier-Stokes sur le tore bidimensionnel. Cette partie du projet est orientée vers une théorie géométrique des écoulements de fluides incompressibles dans l'espace physique.

Mathematics Subject Classification. 34D, 35Q35, 58F, 76.

Received: September 17, 1999. Revised: December 14, 1999.

\section{INTRODUCTION}

The Taylor vector fields, or simply the Taylor fields, are referred to the divergence-free vector fields $v_{n m}$ on the two-dimensional torus $M=\mathbb{T}^{2}=\mathbb{R}^{2} /(2 \pi \mathbb{Z})^{2}$ defined by

$$
v_{n m}=\left(\begin{array}{ll}
m \cos n x_{1} \cos m x_{2}, n \sin n x_{1} \sin m x_{2}
\end{array}\right),
$$

where $n, m \geq 1$ are integers. The Taylor vortices are referred to the periodic structures of the phase diagram of the Taylor fields illustrated by Figure 2.2.

It is easy to see that the Taylor fields are Hamiltonian vector fields whose Hamiltonian functions (or the stream functions) are given by $H_{n m}\left(x_{1}, x_{2}\right)=\cos n x_{1} \cdot \sin m x_{2}$. By the Hodge decomposition, Hamiltonian vector fields on the torus as well as in a general 2-manifold do not exhaust all divergence-free vector fields.

The study of the Taylor vortices is originated in Taylor's 1923 [49]. In fact, such periodic structure appears in many problems of mathematics and physics. The Taylor vortex type of periodic structures appear also in the solutions of many partial differential equations; see Bensoussan, Lions and Papanicolaou [4]. We would like to

\footnotetext{
Keywords and phrases. Divergence-free vector fields, Hamiltonian vector fields, Taylor vortices, Navier-Stokes equations, block structure, block structural stability, structural evolution.

* Dedicated to Roger Temam for his 60th birthday

1 Department of Mathematics, Sichuan University, Chengdu, P.R. China.

2 Department of Mathematics, Indiana University, Bloomington, IN 47405. email: showang@indiana.edu
} 
mention recent very interesting paper by Fannjiang and Papanicolaou [11] studying weak molecular diffusion in the presence of periodic forced convection.

The main objective of this article is to study the structural evolution/transitions of the Taylor vortices with either divergence-free vector fields or Hamiltonian vector fields perturbations. As we know, divergence-free vector fields appear in many fields of mathematical physics. Two most important examples of divergence-free vector fields are the velocity field of an incompressible fluid flows, and the super-current of super-conducting materials.

The main motivation of this and the accompanying articles is to develop a geometric theory of two-dimensional incompressible fluid flows in the physical spaces. The motion of an incompressible fluid is governed by the Navier-Stokes (or Euler) equations, which form an infinite dimensional dynamical system; see among many others $[6-8,18,20-27,34,50]$. From the Lagrangian point of view, the velocity field $v$, which is a solution of the Navier-Stokes equations, determines the dynamics of the fluid particles in the physical space the fluid occupies. To study the geometrical/topological structure of two-dimensional fluid flows in the physical spaces, we adopt a philosophy including the following main aspects/directions:

1. to develop a general (global) geometrical/topological theory of the velocity vector field $v(\cdot, t)$ at each time instant, treating the time $t$ as a parameter, and then

2. allowing the time variable to change, to study the structural transitions of the velocity field $v$.

The study along the first direction was initialized in $[29,30,32,33]$. The main objective in this direction is to establish a geometrical/topological theory for divergence-free vector fields on general two-dimensional compact manifolds with or without boundary. There are connections between the study in this direction and the theory of measured foliations introduced by Thurston [52] although the motivations are different. There are extensive studies by geometers and topologists for the measured foliations as well as for the closely related quadratic differentials; see Fathi and Laudenbach and Poénaru [10], Thurston [52], Strebel [9], and the references therein. In particular, the connections between the dynamics of divergence-free vector fields and measured foliations will be addressed in a forthcoming article [32].

One main result we proved in $[29,33]$ is a global structural stability theorem of divergence-free vector fields, providing necessary and sufficient conditions for structural stability of divergence-free vector fields. The study of structural stability has been one of the main driving force behind much of the development of dynamical systems theory; see among many others the work of Peixoto [40], Smale [47], Palis and de Melo [38], Pugh [41], Shub [42], and the references therein. We are interested in the structural stability of a divergence-free vector field with perturbations of divergence-free vector fields. We call this notion of structural stability the incompressibly structural stability or simply structural stability. Notice that the divergence-free condition changes completely the general features of structurally stable fields as compared to the situation when this condition is not present. The latter case was studied in a classical paper of Peixoto [40]. The conditions for structural stability and genericity in the Peixoto theorem are: (i) the field can have only a finite number of singularities and closed orbits (critical elements) which must be hyperbolic; (ii) there are no saddle connections; (iii) the non wandering set consists of singular points and closed orbits.

The necessary and sufficient conditions for a divergence-free vector field we obtain in $[29,33]$ are: (1) $v$ is regular; (2) all interior saddle points of $v$ are self-connected; and (3) each boundary saddle point is connected to boundary saddles on the same connected component of the boundary. The first condition here requires only regularity of the field and so it does not exclude centers which are not hyperbolic and excluded by (i) above. The second condition is of a completely different nature than the corresponding one in the Peixoto theorem. Namely, condition (ii) above excludes the possibility of saddle connections. In contrast, (2) amounts to saying that all interior saddles are self-connected! Namely, the interior saddles occur in graphs whose topological form is that of the number 8 , being the singularities themselves hyperbolic.

Moreover, a direct consequence of the Peixoto structural stability theorem and the above theorem is that no divergence-free vector field is structurally stable under general $C^{r}$ vector fields perturbations. Such a drastic change in the stable configurations is explained by the fact that divergence-free fields preserve volume and so attractors and sources can never occur for these fields. In particular, this makes it natural the restriction that 
saddles in the boundary must be connected with saddles in the boundary on the same connected component, in the third condition.

The study in the second direction aims in particular at the connections between the solutions of the NavierStokes (or Euler) equations and the dynamics of the velocity fields in the physical space. Progress has also been made in this direction recently in [31], where we proved that for any external forcing in an open and dense subset of $C^{\alpha}(T M)(0<\alpha<1)$, all steady state solutions of the two-dimensional Navier-Stokes equations are structurally stable.

In this article, we classify the structure and its transitions/evolution of the Taylor vortices under small perturbations of either the Hamiltonian vector fields or divergence-free vector fields on the two-dimensional torus. The main ideas and results of this article include the following aspects:

1. A concept called block structure of Hamiltonian vector fields is introduced.

2. The phase structure and its transitions of the Taylor vortices are fully classified in terms of the block structure.

3. Both Hamiltonian and non-Hamiltonian methods of breaking saddle connections of unstable divergencefree vector vector fields are introduced.

4. The structure and its transition of the Taylor vortices are also discussed in the context of the solutions of the Navier-Stokes equations of an incompressible fluid.

Although most discussions in this article are restricted to the Taylor fields, it is hoped that the method and the ideas presented will be useful in understanding the structure and its transitions of other relevant physical problems.

\section{Preliminaries}

Let $M$ be a two-dimensional $C^{r+1}(r \geq 1)$ compact orientable manifold with a natural symplectic structure of the Riemannian area. Let $C^{r}(T M)$ be the space of all $C^{r}$ vector fields on $M$. If $r=k+\alpha$ with $k \geq 0$ an integer and $0<\alpha<1$, then $v \in C^{k}(T M)$ and all derivatives of $v$ up to order $k$ and $\alpha$-Hölder continuous.

A vector field $v \in C^{r}(T M)$ is called regular if all singular points of $v$ are non-degenerate. We set

$$
\begin{aligned}
& D^{r}(T M)=\left\{v \in C^{r}(T M) \mid \operatorname{div} v=0\right\} \\
& D_{0}^{r}(T M)=\left\{v \in D^{r}(T M) \mid v \text { is regular }\right\} \\
& H^{r}(T M)=\left\{v \in D^{r}(T M) \mid v \text { is a Hamiltonian vector field }\right\},
\end{aligned}
$$

where div is the divergence operator on $M$.

Let $v \in D^{r}(T M)$ be a divergence-free vector field, then it is easy to see that (see $\left.[29,33]\right)$ :

1. A non-degenerate interior singular point of $v$ must be either a saddle or a center, and a non-degenerate boundary singular point must be a saddle point;

2. The set of all closed orbits of $v$ is open.

Based on these basic properties of divergence-free vector fields, we have the following definitions.

Definition 1.1. Consider a divergence-free vector field $v \in D^{r}(T M)$.

1. Let $p \in M$ be a center of $v$, then there is an open neighborhood $C$ of $p$, such that for any $x \in C(x \neq p)$, the orbit $\Phi(x, t)$ is closed. The largest neighborhood $C$ of $p$ of this type is called a circle cell of $p$.

2. Let $B \subset M$ be an open set, such that for any $x \in B$, the orbit $\Phi(x, t)$ is closed, and any connected component $\Sigma$ of $\partial B$ is not a single point. Then $B$ is called a circle band of $v$.

3. A closed domain $\Omega \subset M$ (i.e. $\mathrm{cl} \stackrel{\circ}{\Omega}=\Omega$ ) is called an ergodic set of $v \in D^{r}(T M)$ if for any $x \in \Omega$ with the $\omega$-limit set $\omega(x)$ not a singular point of $v, \omega(x)=\Omega$.

4. An orbit with its end points is called a saddle connection if its $\alpha$ and $\omega$ limit sets are saddle points. Moreover, a saddle connection $\gamma$ is called a self saddle connection if $\alpha(\gamma)=\omega(\gamma)$. 
Then we have the following structural classification and stability theorems of Hamiltonian vector fields (see $[32])$.

Theorem 1.2. (Structural Classification Theorem). Let $v \in H^{r}(T M)(r \geq 1)$ be regular. Then the phase diagram of $v$ consists of a finite number of connected components of the following types:

1. circle cells and circle bands, and

2. saddle connections.

Theorem 1.3. (Structural Stability) A Hamiltonian vector field $v \in H^{r}(T M)(r \geq 1)$ is structurally stable under Hamiltonian vector field perturbations if and only if

1. $v$ is regular; and

2. all saddle connections are self saddle connections.

Moreover, the set $H_{s}^{r}(T M)$ of all $C^{r}$ structurally stable Hamiltonian vector fields is open and dense in $H^{r}(T M)$.

Remark 1.4. As we know, Hamiltonian vector fields are divergence-free, but in general do not exhaust all divergence-free vector fields. For a divergence-free vector field, the phase diagram may also contain ergodic sets in addition to circle cells, circle bands and saddle connections in the structural classification theorem. See [32] for details.

\section{BLOCK STRUCTURE}

\subsection{Block structure of Hamiltonian vector fields}

Hereafter, we always denote by $M=\mathbb{T}^{2}$. By the Hamiltonian structural classification and stability theorems, we see that each saddle point of a stable Hamiltonian vector field has exactly two saddle self-connections. Obviously, each saddle self-connection is a simple closed curve.

Definition 2.1. Let $v$ be a stable Hamiltonian vector field on a torus $\mathbb{T}^{2}$, and $Q \subset \mathbb{T}^{2}$ be an invariant set of $v$.

1. $Q$ is called a $D$-block if $Q$ is homeomorphic to an open disk such that $\partial Q$ is a saddle self-connection of a saddle point $p \in \partial Q$, and the other saddle self-connection of $p$, as a closed chain, is not homological to zero in $H_{1}\left(\mathbb{T}^{2}\right)$;

2. $Q$ is called an $S$-block if $Q$ is homeomorphic to an open disk such that $\partial Q$ consists of two saddle selfconnections of a saddle point $p \in \partial Q$;

3. $Q$ is called a $T$-block if $Q$ is a closed domain such that the interior $\stackrel{\circ}{Q}$ does not contain singular points of $v$, and $\mathbb{T}^{2}-Q$ consists of $D$ and $S$-blocks of $v$.

In the above definition, the letters $D, S$ and $T$ stand for disk, sphere and torus respectively; see Figure 2.1 for schematic pictures of the $D, S$ and $T$-blocks.

Definition 2.2. Let $Q \subset M$ be a circle cell of $v \in D^{r}(T M)$. We call that $Q$ has a right-hand orientation (resp. a left-hand orientation) if the interior of $Q$ is on the left side of $\partial Q$ (resp. on the right side of $\partial Q$ ), traveling in the direction of the orbits of $v$ on $\partial Q$,

The right-hand and left-hand orientations for the $D$ and $S$-blocks of a stable Hamiltonian vector field $v$ on $\mathbb{T}^{2}$ can be defined in the same fashion.

The Hamiltonian structural stability theorem tells us that a stable Hamiltonian vector field $v$ on $\mathbb{T}^{2}$ has a block decomposition as follows

$$
\mathbb{T}^{2}=\Omega+\cup_{i=0}^{I} D_{i}+\cup_{k=1}^{K} A_{k} \text { with } D_{0}=\emptyset .
$$

Here $\Omega$ is a unique $T$-block, $D_{i}(0 \leq i \leq I)$ are $D$-blocks and $A_{k}(1 \leq k \leq K)$ are $S$-blocks of $v$, which are topologically equivalent to a block structure as shown in Figure 2.1 below.

The following theorem shows that each stable Hamiltonian vector field on $\mathbb{T}^{2}$ has exactly one $T$-block, at least two $S$-blocks and possibly zero $D$-blocks. 


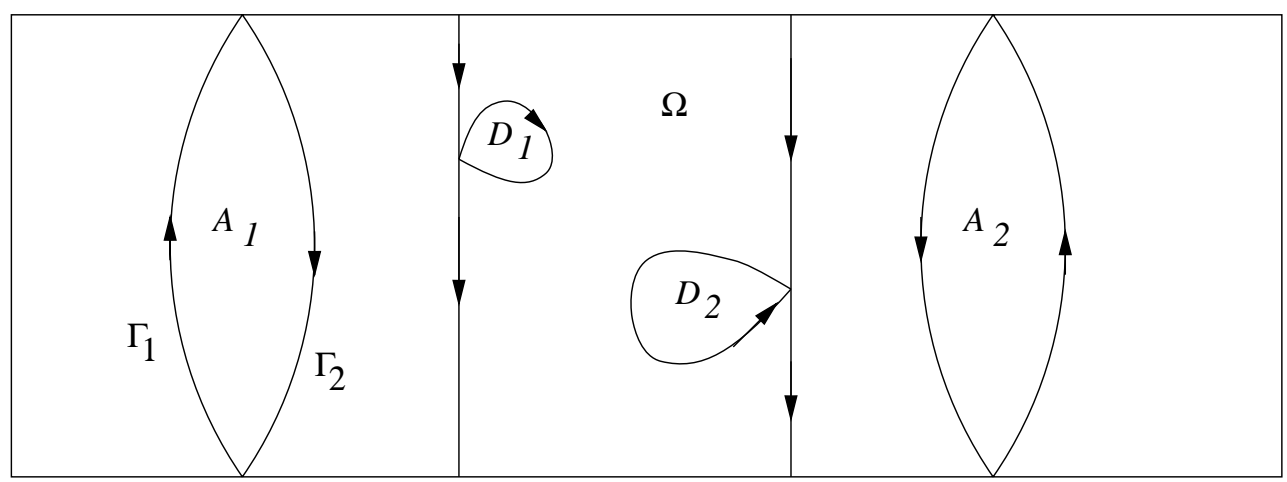

Figure 2.1. $A_{1}$ and $A_{2}$ are two $S$-blocks with left-hand and right-hand orientations respectively, $D_{1}$ and $D_{2}$ are $D$-blocks with left-hand and right-hand orientations, and $\Omega$ is a $T$-block.

Theorem 2.3. A Hamiltonian vector field $v \in H^{r}(T M)$ is structurally stable in $H^{r}(T M)$ if and only if $v$ has a block decomposition as in (2.1). Moreover, for any stable Hamiltonian vector field $v \in H_{s}^{r}(T M)$ on $M=\mathbb{T}^{2}$,

1. $v$ has at least two $S$-blocks and the number $K$ of the $S$-blocks of $v$ is even, i.e. $K=2 m(m \geq 1)$. Moreover half of the $S$-blocks have right-hand orientation and the other half have left-hand orientation;

2. $v$ has exactly one $T$-block.

Proof. Thanks to the structural classification and stability theorems, it is easy to see that a Hamiltonian vector field $v \in H^{r}(T M)$ having block decomposition as in (2.1) is a necessary and sufficient condition for $v$ being structurally stable in $H^{r}(T M)$.

Hence it suffices to prove Assertions 1 and 2. To this end, let $v \in H_{s}^{r}(T M)$ be a stable Hamiltonian vector field. Notice that the boundary of each $S$-block $A_{k}$ has two saddle self-connections, say $\Gamma_{1}$ and $\Gamma_{2}$ as shown in Figure 2.1, and the orientation of closed orbits in $\Omega$ near $\Gamma_{1}$ are reverse to the orientation of the closed orbits in $\Omega$ near $\Gamma_{2}$. Obviously, a $D$-block does not reverse the orientation of the orbits in $\Omega$ Therefore the number of $S$-blocks of $v$ must be even, i.e. $K=2 m$, and $m$ of these $S$-blocks have right-hand orientation, and the other $m S$-blocks have left-hand orientation.

Now we show that $m \geq 1$. Assume otherwise, i.e. $m=0$, then the $T$-block $\Omega$ of $v$ is a compact manifold with boundary, which consists of closed orbits and saddle self-connections of $v$. Let $v=d H^{\#}$, where $H \in C^{r+1}(M)$ is a Hamiltonian function. Then all orbits in $\stackrel{\circ}{\Omega}$ are level lines, and there is an orbit $\gamma \subset \stackrel{\circ}{\Omega}$ such that $H$ takes the maximum value on $\gamma$.

For any point $q \in \gamma$, we take an orthogonal coordinate system $\left(x_{1}, x_{2}\right)$ on the tangent space $T_{q}\left(\mathbb{T}^{2}\right)$ with the origin at $q$ and the $x_{1}$-axis tangent to $\gamma$ at $q$. Since $\gamma$ is a level line of $H$, and $H$ takes the maximum value at $q \in \gamma$ along with $x_{2}$-axis, we have

$$
\frac{\partial H(q)}{\partial x_{1}}=0, \quad \frac{\partial H(q)}{\partial x_{2}}=0
$$

which implies that $v=0$ on $\gamma$, contrary to $v$ being regular.

The proof is complete.

In this article, we study the structural evolution of the Taylor fields on the Torus under the perturbation of either Hamiltonian vector fields or general divergence-free vector fields.

Definition 2.4. 1. Let $v_{1}, v_{2} \in H_{s}^{r}(T M)$ with block decompositions as in (2.1). We say that $v_{1}$ and $v_{2}$ have isomorphic block decompositions if there exists a homeomorphism $\varphi: M \rightarrow M$ such that $\varphi$ takes the $D, S$ and $T$-blocks of $v_{1}$ to the $D, S$ and $T$-blocks of $v_{2}$ respectively, preserving the $D$ and $S$-block orientations. 
2. The set of the stable Hamiltonian vector fields with isomorphic block decompositions is called a block isomorphic class, and the set of the topologically equivalent Hamiltonian vector fields is called a topologically equivalent class.

\subsection{Block structure of divergence-free vector fields}

We start with block stability theorem for divergence-free vector fields introduced in [32].

Definition 2.5. A regular $v \in D_{0}^{r}(T M)$ is called a basic vector field if $M$ can be decomposition into invariant blocks as $M=\Omega \cup_{k=0}^{K} A_{i}$ with empty intersections between them, $A_{0}=\emptyset$ and such that

1. each $A_{k}$ is an open flow-invariant sub-manifold which is homeomorphic to an open disk, and $\Omega$ is a compact invariant sub-manifold with genus one, and

2. for each $1 \leq k \leq K,\left.v\right|_{A_{k}}$ is a self-connection vector field,

3. $v$ has only one saddle point in each $\partial A_{k}(1 \leq k \leq K)$, and has no singular point in $\stackrel{\circ}{\Omega}$.

We denote by $D_{B}^{r}(T M)$ the set of all $C^{r}$ basic vector fields.

Theorem 2.6. (Block Stability Theorem [32]) Let $v \in D_{B}^{r}(T M)$ be a basic vector field with block decomposition $M=\Omega \cup_{k=0}^{K} A_{k}$. Then there exists a neighborhood $\mathcal{O} \subset D^{r}(T M)$ of $v$ such that

1. each $v_{1} \in \mathcal{O}$ has block decomposition $M=\Omega^{(1)} \cup_{i=0}^{K} A_{i}^{(1)}$ which is isomorphic to that of $v$;

2. $\left.v\right|_{A_{i}}$ is topologically equivalent to $\left.v_{1}\right|_{A_{i}^{(1)}}$ for $v_{1} \in \mathcal{O}$;

3. there is a dense set $\widetilde{\mathcal{O}} \subset \mathcal{O}$ such that for any $v_{1} \in \widetilde{\mathcal{O}}, \Omega^{(1)}$ is an ergodic set of $v_{1}$; and

4. $D_{B}^{r}(T M)$ is open and dense in $D^{r}(T M)$.

The differences between the block structures of the divergence-free vector fields and the Hamiltonian vector fields are as follows: 1 ). there are no $S$-blocks in the block structure of basic divergence-free fields; 2) the $T$-block $\Omega$ of a basic divergence-free vector field is a manifold, which may be an ergodic set. Furthermore, it is easy to see that no divergence-free vector fields on the torus is structurally stable under the perturbation in $D^{r}(T M)$, due essentially to the existence of ergodic sets.

\subsection{The Taylor fields}

Let

$$
M=\mathbb{R}^{2} /(2 \pi \mathbb{Z})^{2}
$$

The Taylor fields are defined by

$$
v_{n m}=\left(\begin{array}{lll}
m \cos n x_{1} & \cos m x_{2}, n \sin n x_{1} \sin m x_{2}
\end{array}\right)
$$

where $n, m \geq 1$ are integers. The Hamiltonian functions (or the stream functions) of (3.1) are given by

$$
H_{n m}\left(x_{1}, x_{2}\right)=\cos n x_{1} \sin m x_{2} .
$$

From (2.2), it is easy to see that each Taylor field $v_{n m}$ has $4 n m$ saddle points and $4 n m$ centers. The saddle points of $v_{n m}$ are

$$
\left(\frac{2 k_{1}+1}{2 n} \pi, \frac{k_{2}}{m} \pi\right), \quad k_{1}=0,1, \cdots, 2 n-1 ; \quad k_{2}=0,1, \cdots, 2 m-1
$$

and the centers of $v_{n m}$ are

$$
\left(\frac{k_{1}}{n} \pi, \frac{2 k_{2}+1}{2 m} \pi\right), k_{1}=0,1, \cdots, 2 n-1 ; \quad k_{2}=0,1, \cdots, 2 m-1 .
$$




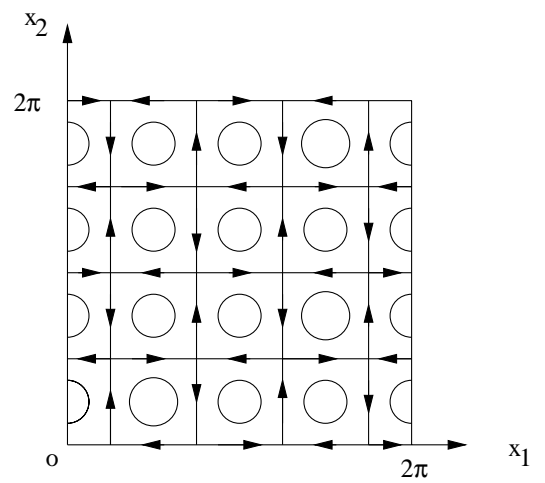

FiguRE 2.2

For instance, the phase diagrams of the Taylor fields $v_{22}$ are shown in Figure 2.2.

By the structural stability theorem, the Taylor fields (2.2) are unstable because they are not self-connection fields. Since the set of all stable Hamiltonian vector fields $H_{s}^{r}(T M)$ is open and dense in $H^{r}(T M)$, under a perturbation in $H^{r}(T M)$, a Taylor field $v_{n m}$ will be transformed into a stable Hamiltonian vector field. We consider the problem what and how many types of stable Hamiltonian vector fields one can obtain by perturbing slightly the Taylor field $v_{n m}$ with either Hamiltonian fields or general divergence-free vector fields. The key procedure is to break saddle connections of the Taylor vortices. In next section, we shall introduce some methods of breaking saddle connections, and then we will study the questions raised for the Taylor fields.

\section{Methods of BREAKIng SAdDLE CONNECTIONS}

In this section we introduce some methods to break saddle connections of the Taylor vortices. The main tool is the tubular flow introduced in the following lemma.

Lemma 3.1. Let $L \subset M$ be a $C^{r}$ simple closed curve, and $B \subset M$ be an open annulus with $L$ being one connected component of $\partial B$.

1. There exists a divergence-free vector field $v \in D^{r}(T M)$ with

$$
\left.v\right|_{M-B}=0,\left.\quad v\right|_{B} \neq 0 .
$$

2. The vector field (3.1) can be chosen as a Hamiltonian vector field if and only if $L \subset M$, as a closed chain, is homological to zero.

The proof of this lemma is given in [32].

\subsection{Hamiltonian breaking method}

We know the local structure of the flow pattern of a Taylor field $v_{n m}$ is as shown in Figure 3.1(a) below.

For a saddle point $q$ which is connected with other saddle points, we set an open annulus $B$ with $\partial B$ retractable to $q$ in $M$, and take a Hamiltonian vector field $w$ as in Lemma 3.1 (see Fig. 3.1(a)). Then for any $\lambda>0$ sufficiently small the pattern Figure 3.1(a) of $v$ transforms to the pattern in Figure 3.1(b) of $v+\lambda w$. We call this method Type I Local Breaking Method at the saddle point $q$.

Notice that by reversing the orientation of $w$, the saddle points are connected slightly differently as illustrated by Figures 3.1 and 3.2 . 


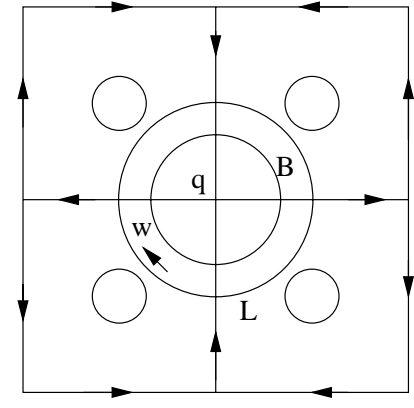

(a)

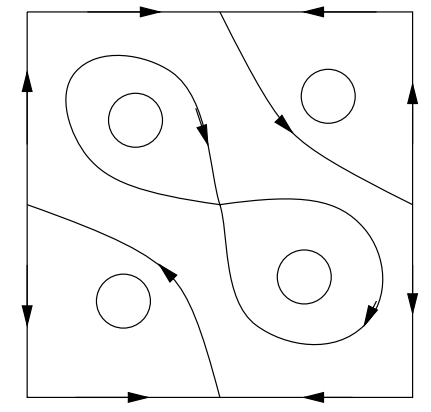

(b)

Figure 3.1

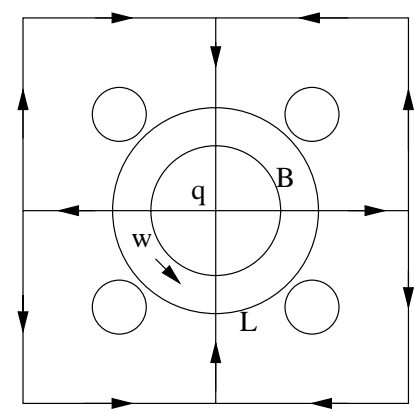

(a)

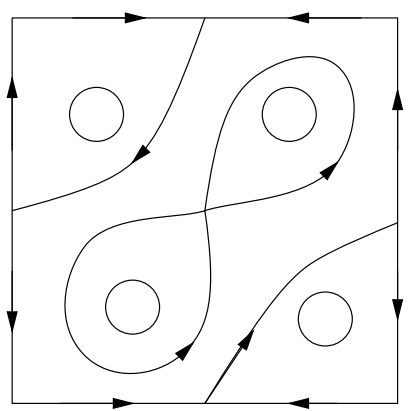

(b)

FigURE 3.2

Remark 3.2. The perturbation field $w$ can be constructed analytically as follows. Let $0<r_{1}<r_{2}$ be two given small numbers and $\psi \in C^{\infty}[0, \infty)$ such that

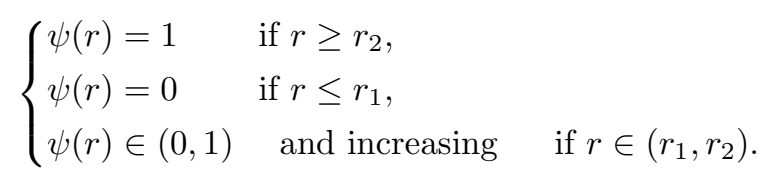

Then for $0<r_{1}<r_{2}$ small, the divergence-free vector fields

$$
\left\{\begin{array}{l}
w_{1}=\operatorname{curl} \psi\left(\left|\left(x_{1}, x_{2}\right)-\left(x_{1}^{0}, x_{2}^{0}\right)\right|\right) \\
w_{2}=\operatorname{curl}\left[1-\psi\left(\left|\left(x_{1}, x_{2}\right)-\left(x_{1}^{0}, x_{2}^{0}\right)\right|\right)\right]
\end{array}\right.
$$

are typical examples of the perturbation field in Figures 3.1 and 3.2 respectively. Here $\left(x_{1}^{0}, x_{2}^{0}\right)$ is the coordinate of $q, r_{1}$ and $r_{2}$ the radii of the inner and outer circles of the circle band $B$.

\subsection{Non-Hamiltonian breaking method}

As we know the Hamiltonian vector fields do not exhaust all divergence-free vector fields. We now construct two typical families of non-Hamiltonian divergence-free vector fields, and apply them to the flow structure of the Taylor vortices. 


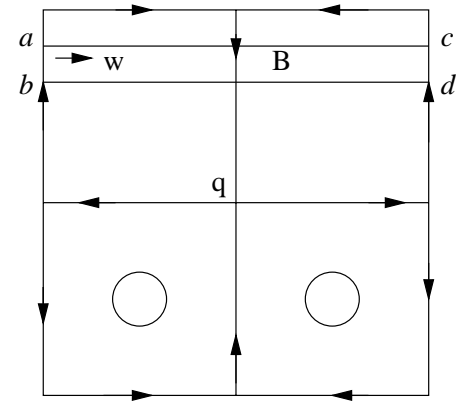

(a)

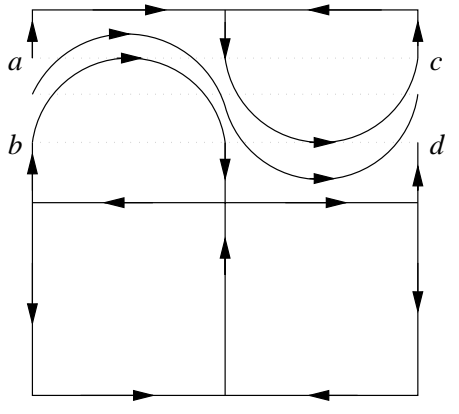

(b)

Figure 3.3. Type II Local Breaking Method. The segment $a b$ (resp. $c d$ ) in (b) is the same as $a b$ (resp. $c d$ ) in (a). In (b), any orbit passing through a point in the open segment $a b$ is periodic.

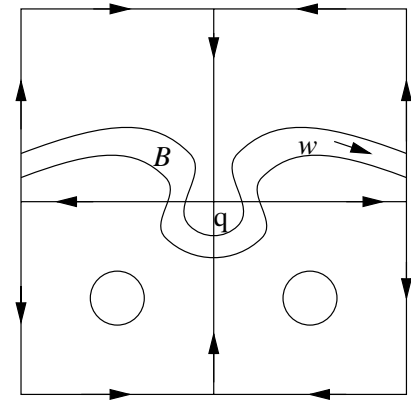

(a)

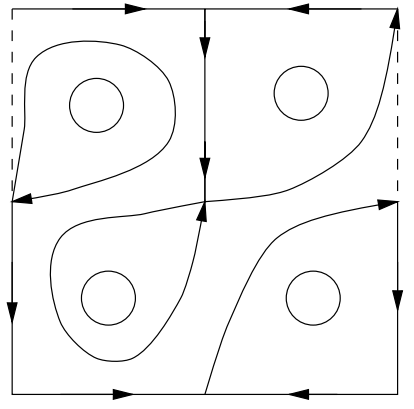

(b)

Figure 3.4. Type III Local Breaking Method.

Consider again the saddle point $q$, which is connected by four saddle connections. In the Hamiltonian case, the closed curve $L$ with zero homology has to be chosen to intersect each of the four saddle connections at least one time. In the non-Hamiltonian case, we can chose $L$ to intersect either one or three of the four saddle connections as shown in Figures 3.3(a) and 3.4(a) below. Then for any $\lambda>0$ sufficiently small the pattern Figure 3.3(a) (resp. Fig. 3.3(a)) of $v$ transforms to the pattern in Figure 3.3(b) (resp. Fig. 3.3(b)) of $v+\lambda w$. We call the method illustrated in Figure 3.3 Type II Local Breaking Method at the saddle point q, and the method corresponding to Figure 3.4 Type III Local Breaking Method at the saddle point q.

\section{Structural evolution of the Taylor fields with Hamiltonian PERTURBATIONS}

\subsection{Structural evolution of the general Taylor fields}

Our first main result is the following theorem on the structural evolution of the general Taylor field $v_{n m}(1 \leq$ $m \leq n)$. In next subsection, we shall study the detailed structural evolution of $v_{11}$.

Theorem 4.1. Let $v_{n m}(1 \leq m \leq n)$ be a Taylor field. Then for any sufficiently small neighborhood $\mathcal{O} \subset$ $H^{r}(T M)$ of $v_{n m}$ and $v \in \mathcal{O} \cap H_{s}^{r}(T M)$, we have 


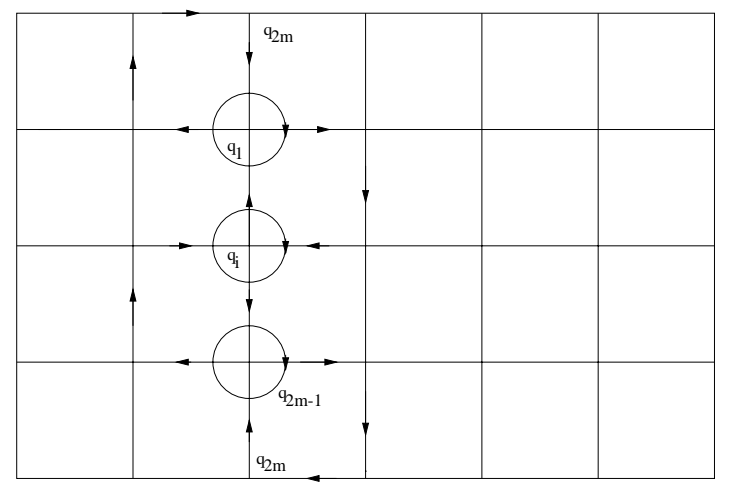

Figure 4.1. Single circles are used to denote the tubular flows near saddle points $q_{j}$ here as well as in figures hereafter.

1. the number $K=2 R$ of the $S$-blocks of $v$ satisfies

$$
1 \leq R \leq n
$$

2. for each $R(1 \leq R \leq n)$ there is a $v \in \mathcal{O} \cap H_{s}^{r}(T M)$ such that $v$ has exactly $2 R S$-blocks;

3. in each $S$-block of $v \in \mathcal{O} \cap H_{s}^{r}(T M)$, there are at least $2 m$ centers (or $2 m-1$ saddle points) of $v$; and

4. when $m=1$, all circle cells in right hand $D$ and $S$ blocks (resp. in left hand $D$ and $S$ blocks) are right hand orientation (resp. left hand orientation).

Proof. Step 1. Let $L \subset M$ be an invariant curve of a vector field $v$, and $p_{1}, \cdots, p_{k} \in L$ be the saddle points of $v$ on $L$. These saddle points divide $L$ into $m$ segments $\gamma_{1}, \cdots, \gamma_{m}$, which are orbits of $v . L$ is called an orbit curve if the limit sets of the orbit $\gamma_{i}(1 \leq i \leq m)$ satisfy

$$
\omega\left(\gamma_{i}\right)=\alpha\left(\gamma_{i+1}\right), \quad 1 \leq i \leq m-1 .
$$

For example, the boundary $\Gamma_{1} \cup \Gamma_{2}$ of an $S$-block $A_{k_{1}}$ in Figure 2.1 is an orbit curve. If a closed orbit curve $L$, as a closed chain, is homological to zero, then there must be an open set $A \subset M$ such that $\partial A=L$, and we say that $L$ is retractable if the closure $\bar{A}$ is retractable to a point in $M$.

It is easy to prove the following lemma; we omit the details of the proof.

Lemma 4.2. Let $v_{n}, v \in D^{r}(T M)$ be regular with $v_{n} \rightarrow v(n \rightarrow \infty)$, and $L_{n}$ be a closed orbit curve of $v_{n}$. Then there are a closed orbit curve $L$ of $v$ and a subsequence still denoting by $L_{n}$ such that $L_{n} \rightarrow L(n \rightarrow \infty)$, and

1. $L$ is homological to zero if and only if $L_{n}$ are homological to zero.

2. If $L$ is retractable, then $L_{n}$ are retractable.

3. Let $\partial A_{n}=L_{n}$ and $\partial A=L$ and $\bar{A}_{n} \rightarrow \bar{A}$. Then for any $n$ sufficiently large the number of centers of $v_{n}$ in $A_{n}$ equals to the number of centers of $v$ in $A$.

Now we come back to prove Assertions 1-4. Since a Taylor field $v_{n m}(1 \leq m \leq n)$ has $4 n m$ centers, and from the flow pattern of $v_{n m}$ (see Fig. 2.1) we can see that each open set $A$ enclosed by a closed non-retractable orbit curve must contain at least $2 m$ centers. Hence Assertions 1 and 3 follow from Lemma 4.2.

Step 2. Proof of Assertion 2. We proceed in a few cases as follows.

Case $R=n$ : We first construct vector fields in $\mathcal{O} \cap H_{s}^{r}(T M)$ with exactly $2 R=2 n S$-blocks. Consider a non-retractable closed orbit curve $L$ enclosing exactly $2 m$ circle cells, and having exactly $2 m$ self-intersecting points $q_{1}, \cdots, q_{2 m}$. 


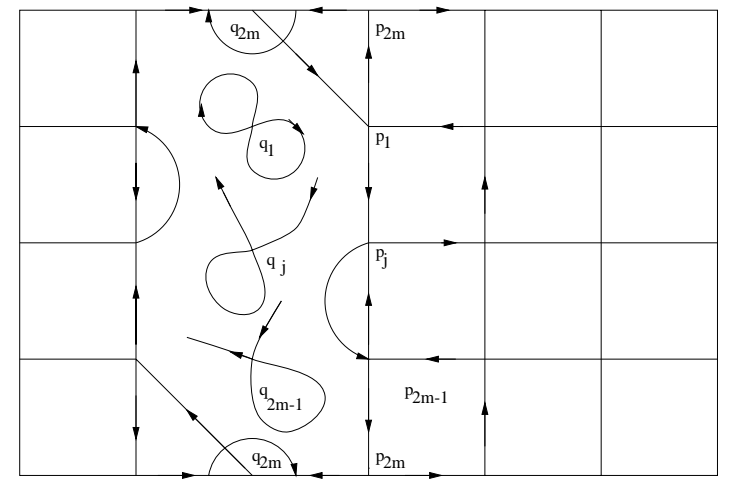

FiguRE 4.2. Single circles are used to denote the tubular flows near saddle points $q_{j}$ here as well as in figures hereafter.

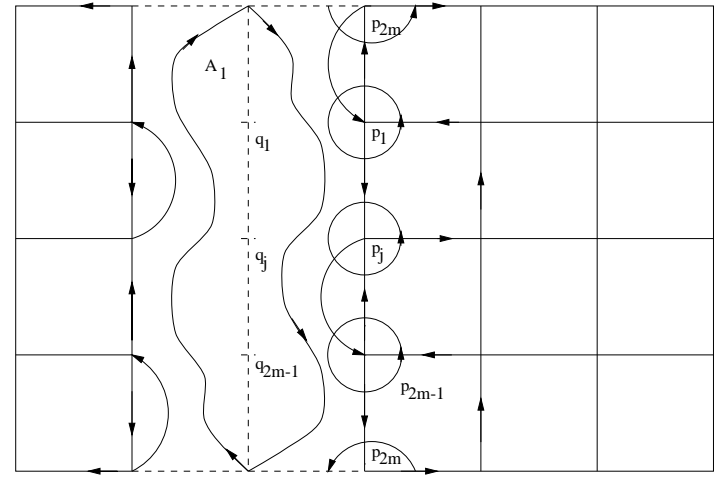

Figure 4.3

Then we apply the Type I Local Breaking Method with clockwise tubular flows described at the end of the previous section to the Taylor field at the saddle points $q_{i}(1 \leq i \leq 2 m-1)$; see Figure 4.1. The flow pattern in Figure 4.1 transforms to the flow pattern in Figure 4.2. Next apply again the Type I Local Braking Method near the saddle point $q_{2 m}$ still with clockwise tubular flow, the flow pattern transforms into that in Figure 4.3, which contains one $S$-block $A_{1}$.

Next we consider a non-retractable closed orbit curve $\tilde{L}$ enclosing exactly $2 m$ circle cells, and with $2 m$ selfintersecting saddle points saddle points $p_{1}, \cdots, p_{2 m-1}, p_{2 m}$ as shown in Figure 4.3. Applying the Type I Local Breaking Method with counter-clock-wise tubular flows near these saddle points, we obtain another $S$-block, which we call $A_{2}$, enclosing the saddle points $p_{1}, \cdots, p_{2 m-1}$; see Figure 4.4. Inductively, in exactly $2 n$ steps, the flow pattern in Figure 4.2 will be transfered to the flow pattern having exactly $2 n S$-blocks.

Case $R=1$ : We construct vector fields in $\mathcal{O} \cap H_{s}^{r}(T M)$ with exactly $2 R=2 S$-blocks.

As in the previous case, we obtain an $S$-block as shown in Figure 4.3. Then, instead of the counterclockwise tubular flows, we apply the Type I Local Breaking Method with clockwise tubular flows near the saddle points $p_{1}, \cdots, p_{2 m-1}, p_{2 m}$. We obtain then $2 m$ D-blocks as shown in Figure 4.5.

Repeating this procedure $2 n-2$ times and then applying the Type I Local Breaking Method to the last non-retractable orbit curve on the right of the $S$-block $A_{1}$ with clockwise tubular flows, we obtain another $S$-block and all other saddles are connected to a $D$-block as shown in Figure 4.5. 


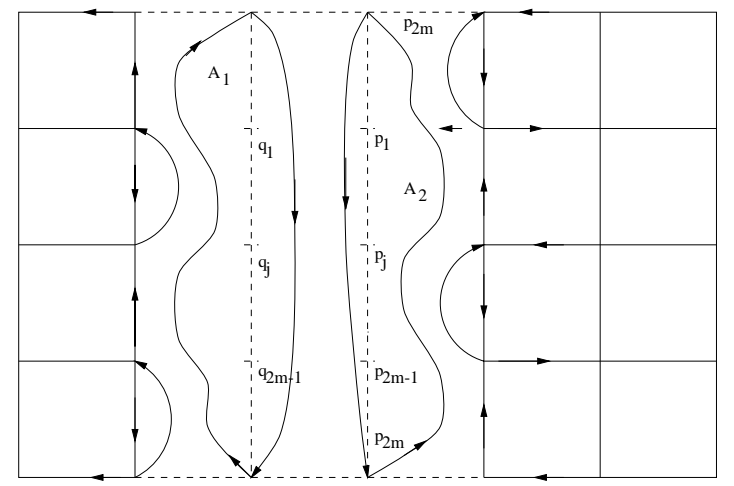

FiguRE 4.4

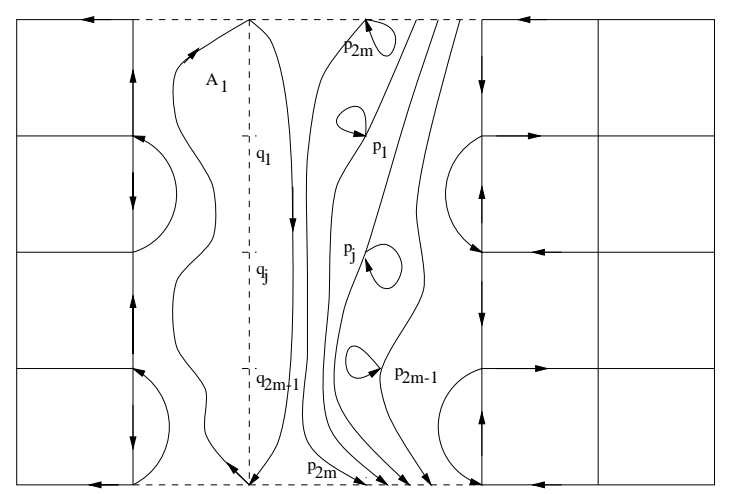

FigURE 4.5

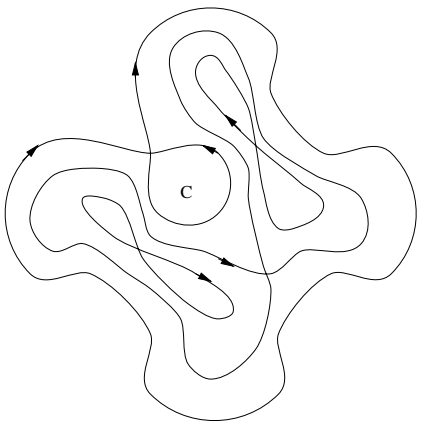

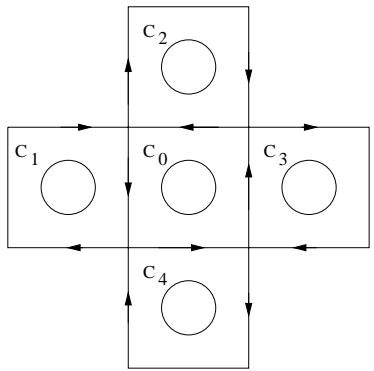

(b)

FigURE 4.6

Case $1<R<n$ : In this case, we construct the fields in $\mathcal{O} \cap H_{s}^{r}(T M)$ having exactly $2 R S$-blocks in two steps. First, we obtain $2 R S$-blocks as in the case where $R=n$; then we break all other saddles into $D$-blocks.

Step 3. Proof of Assertion 4. For any $v \in \mathcal{O} \cap H_{s}^{r}(T M), v$ is self-connection. Since each closed orbit curve of $v_{1 n}$ contains at least four saddle points. Therefore if a left hand $S$-block $A^{-}$contains a right-hand circle cell $C$, then flow pattern adjacent to $C$ must be as illustrated by Figure 4.6(a). 


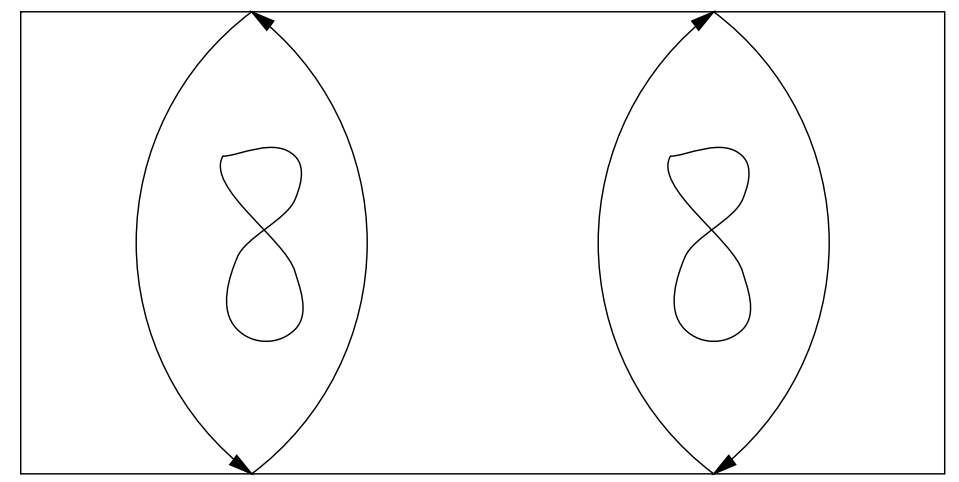

FiguRE 4.7

The flow pattern in Figure 4.6(a) can only be obtained by perturbing the flow pattern in Figure 4.6(b) in the Taylor field. In Figure 4.6(b), there are four different left-hand circle cells $C_{1}, C_{2}, C_{3}$ and $C_{4}$ adjacent to the right-hand circle cell $C_{0}$, corresponding to $C$ in Figure 4.6(a). Obviously there is no such structure in the flow pattern of $v_{1 n}$. Assertion 4) is proved.

The proof is complete.

\subsection{Uniqueness of the structural evolution of $v_{11}$}

When $m=n=1$, we have the following uniqueness result of the structural evolution of the Taylor field $v_{11}$. In next subsection, we shall construct analytically the perturbation fields, and will make connections between the solutions of the Navier-Stokes equations.

Theorem 4.3. There is a neighborhood $\mathcal{O} \subset H^{r}(T M)$ of $v_{11}$ such that $\mathcal{O} \cap H_{s}^{r}(T M)$ consists of exactly one topologically equivalent class. The standard phase portrait of the topologically equivalent class $\mathcal{O} \cap H_{s}^{r}(T M)$ is as shown in Figure 4.7.

Proof. By Claims 1), 3) and 4) in Theorem 4.1, each $v \in \mathcal{O} \cap H_{s}^{r}(T M)$ has a block decomposition isomorphic to the standard decomposition

$$
M=\Omega+A^{+}+A^{-},
$$

where $\Omega$ is a $T$-block, $A^{+}$and $A^{-}$are $S$-blocks with right hand and left hand orientations respectively. Therefore, $v$ has a saddle connection diagram isomorphic to that as shown in Figure 4.7. By the Topological Classification Theorem in [32], Theorem 4.3 follows.

\section{Structural evolution of the Taylor Fields With Divergence-Free Vector FIELD PERTURBATIONS}

The structure evolution of the Taylor fields under the perturbation of divergence-free vector fields is quite different. In this section, we only give an example to show the difference.

Due mainly to these differences, under a perturbation in $D^{r}(T M)$ a Taylor field $v_{n m}$ may be transformed into a vector field which has $4 n m D$-blocks, i.e. each center corresponds to a $D$-block. More precisely, for a Taylor field $v_{n m}$ and any sufficiently small neighborhood $\mathcal{O} \subset D^{r}(T M)$ of $v_{n m}$, the number $K$ of the $D$-blocks of $v \in \mathcal{O} \cap D_{B}^{r}(T M)$ satisfies

$$
2 \leq K \leq 4 m n
$$




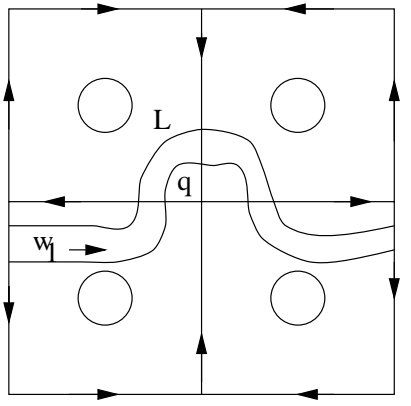

(a)

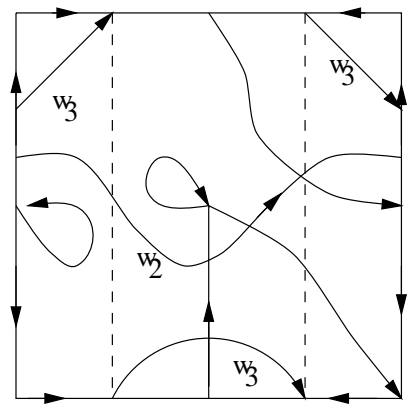

(b)

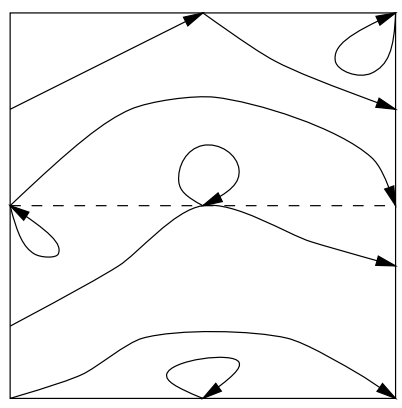

(c)

FiguRE 5.1

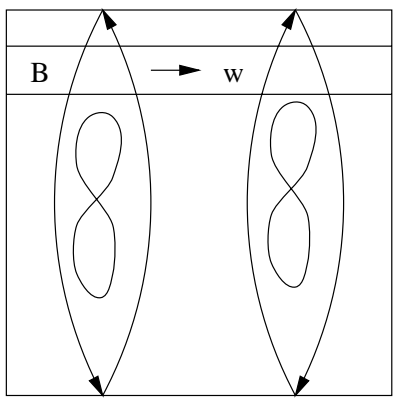

(a)

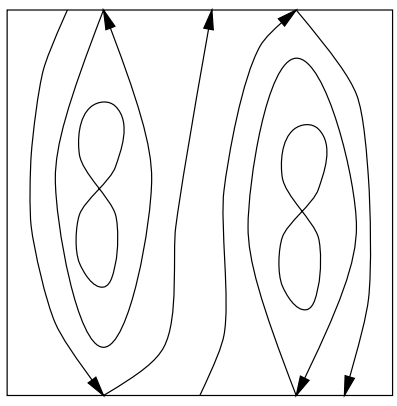

(b)

FIGURE 5.2

and for each $K(2 \leq K \leq 4 m n)$ there are $v \in \mathcal{O} \cap D_{B}^{r}(T M)$ such that $v$ have $K D$-blocks.

We now illustrate this point with the following example.

Consider the Taylor field $v_{11}=\left\{\cos x_{1} \cos x_{2}, \sin x_{1} \sin x_{2}\right\}$, which has the flow pattern as shown in Figure 5.1(a). By Lemma 3.1, we take a tubular incompressible vector field $w_{1}$ as in Figure 5.1(a). Under the perturbation $\lambda_{1} w_{1}$ the flow pattern in Figure 5.1(a) of $v_{11}$ becomes the pattern in Figure 5.1(b) of $v_{11}+\lambda_{1} w_{1}(\lambda>$ $0)$. Then take two tubular incompressible vector fields $w_{2}$ and $w_{3}$ as in Figure 5.1(b). Under the perturbation of $\lambda_{2} w_{2}$ and $\lambda_{3} w_{3}\left(\lambda_{2}>0, \lambda_{3}>0\right)$, the flow pattern (b) will be transformed into the flow pattern (c) of $v_{11}+\lambda_{1} w_{1}+\lambda_{2} w_{2}+\lambda_{3} w_{3}$, which is as desired.

Of course, there are other ways to break the saddle connections of $v_{11}$. For instance, using the Type I Local Breaking Method (with Hamiltonian perturbations), we obtain the flow pattern as shown in Figure 4.7. Then applying the Type II Local Breaking Method as shown in Figure 5.2(a), we obtain the block structure Figure 5.2(b). In this case, the final flow pattern Figure 5.2(b) has a $T$ block and two $D$ blocks, each of which contains two centers and an eight-shaped saddle connection.

In fact, it is easy to see that there is a neighborhood $\mathcal{O} \subset D^{r}(T M)$ of $v_{11}$ such that $\mathcal{O} \cap D_{B}^{r}(T M)$ consists of exactly five topologically equivalent class, including the two given in Figures 5.1(c) and 5.2(b).

\section{Structural evolution of the Taylor fields as solutions OF THE NAVIER-STOKES EQUATIONS}

Since the velocity field of fluid flows is governed by the hydro-dynamical equations, it is necessary to consider the structural evolution of the Taylor fields with the perturbations of the solutions of the Navier-Stokes equations. 
We explore this objective in two aspects: 1) treat the Taylor fields as the steady state solutions of the Navier-Stokes equations, and 2) treat the Taylor fields as initial fields of the time-dependent Navier-Stokes equations.

We start with the following Navier-Stokes equations on the torus $M=\mathbb{T}^{2}=\mathbb{R}^{2} /(2 \pi \mathbb{Z})^{2}$ :

$$
\begin{aligned}
& \frac{\partial u}{\partial t}+u \cdot \nabla u+\nabla p-\triangle u=f, \quad x \in M, \\
& \operatorname{div} u=0, \\
& u\left(x_{1}+2 \pi, x_{2}\right)=u\left(x_{1}, x_{2}+2 \pi\right)=u\left(x_{1}, x_{2}\right), \\
& u(x ; 0)=u_{0}(x) .
\end{aligned}
$$

For any $u \in D^{r}(T M)$, we have the following Fourier expansion:

$$
\left\{\begin{array}{l}
u=\left(u_{1}, u_{2}\right), \\
u_{1}=\sum_{n, m=-\infty}^{\infty} a_{n m} e^{i\left(n x_{1}+m x_{2}\right)}, \\
u_{2}=\sum_{n, m=-\infty}^{\infty} b_{n m} e^{i\left(n x_{1}+m x_{2}\right)}, \\
a_{-n-m}=\bar{a}_{n m} \quad b_{-n-m}=\bar{b}_{n m}, \\
n a_{n m}+m b_{n m}=0 .
\end{array}\right.
$$

By the Hodge decomposition, we have

$$
\left\{\begin{array}{l}
C^{r}(T M)=D^{r}(T M) \oplus G^{r}(T M) \\
G^{r}(T M)=\left\{\nabla \phi \mid \psi \in C^{r+1}(M)\right\} \\
D^{r}(T M)=H^{r}(T M) \oplus \mathcal{H}
\end{array}\right.
$$

Here $\mathcal{H}$ contains all harmonic fields with dimension

$$
\operatorname{dim} \mathcal{H}=2 \text { the first Betti number of } M \text {. }
$$

It is easy to see that

$$
\mathcal{H}=\{u=(a, b) \mid a, b \in \mathbb{R}\}=\mathbb{R}^{2} .
$$

In view of the Fourier expansion, if $a_{00}=b_{00}=0$, then $u$ given by (6.5) is a Hamiltonian field, whose Hamiltonian function is given by

$$
H=-i \sum_{\substack{n, m=-\infty \\ n \neq, m \neq 0}}^{\infty} \frac{1}{m} a_{n m} e^{i\left(n x_{1}+m x_{2}\right)}-i \sum_{m=-\infty}^{\infty} \frac{1}{m} a_{0 m} e^{i m x_{2}}-i \sum_{n=-\infty}^{\infty} \frac{1}{n} b_{n 0} e^{i n x_{1}}
$$

Theorem 6.1. If $u_{0} \in H^{r}(T M), f \in H^{r-2}(T M) \oplus G^{r-2}(T M)(r \geq 2)$, then the solution $u$ of (6.1-6.4) is a one parameter family of Hamiltonian vector fields, i.e. $u(\cdot ; t) \in H^{r}(T M)$ for any $t \geq 0$. 
Proof. Let

$$
\begin{aligned}
& P_{1}: D^{r}(T M) \longrightarrow H^{r}(T M), \\
& P_{2}: D^{r}(T M) \longrightarrow \mathcal{H}, \\
& P_{3}: C^{r}(T M) \longrightarrow G^{r}(T M),
\end{aligned}
$$

be the projection operators.

First we claim that for any

$$
P_{2}[u \cdot \nabla u]=0 \quad \forall u \in D^{r}(T M) .
$$

Indeed it suffices to show that

$$
\int_{\mathbb{T}^{2}} u \cdot \nabla u=0 \quad \forall u \in D^{r}(T M) .
$$

By the Hodge decomposition, let

$$
u=\left(u_{1}, u_{2}\right)=\left(a_{1}+\frac{\partial \psi}{\partial x_{2}}, a_{2}-\frac{\partial \psi}{\partial x_{1}}\right)
$$

where $\psi \in C^{r+1}(M)$, and $a_{1}, a_{2}$ are constants. Then direct computation shows (6.10) holds true; therefore (6.9) holds true.

Now let $u$ satisfy the Navier-Stokes equations (6.1-6.4), and set $u=u_{1}+u_{2}, u_{1}=P_{1} u, u_{2}=P_{2} u$. Then by (6.9) we derive easily

$$
\left\{\begin{array}{l}
\frac{\partial u_{1}}{\partial t}+P_{1}[u \cdot \nabla u]-\triangle u_{1}=P_{1} f \\
\frac{\partial u_{2}}{\partial t}=0 \\
u_{1}(x ; 0)=u_{0}(x) \\
u_{2}(x ; 0)=0
\end{array}\right.
$$

Then $u_{2}(\cdot, t)=0$ for any $t \geq 0$, and the proof is complete.

The steady state case. Let $v_{n m}$ be a steady state solution of the Navier-Stokes equations. Namely,

$$
P_{1}\left[v_{n m} \cdot \nabla v_{n m}\right]-\triangle v_{n m}=P_{1} f
$$

for some $f \in H^{r-2}(T M) \oplus G^{r-2}(T M)(r \geq 2)$. Let $w$ be a another Hamiltonian vector field. Then for any $\lambda>0, v_{n m}+\lambda w$ satisfies

$$
\left\{\begin{array}{l}
P_{1}\left[\left(v_{n m}+\lambda w\right) \cdot \nabla\left(v_{n m}+\lambda w\right)\right]-\triangle\left(v_{n m}+\lambda w\right)=P_{1} f+\lambda \hat{f} \\
\hat{f}=-P_{1}\left[w \cdot \nabla v_{n m}+v_{n m} \cdot \nabla w+\lambda w \cdot \nabla w\right]+\triangle w .
\end{array}\right.
$$

Therefore by perturbing the external forcing $P_{1} f$ with $\lambda \hat{f}(\lambda>0$ small), the evolution of the Taylor vortices (fields) discussed in this article holds true when restricting the perturbation fields to the solutions of the steady state Navier-Stokes equations. 
More precisely, for any $0<\alpha<1$, let

$$
G=L+N: H^{2+\alpha}(T M) \rightarrow H^{\alpha}(T M)
$$

be a map such that for $u \in H^{2+\alpha}(T M)$,

$$
\left\{\begin{array}{l}
L(u)=-\mu \Delta u \\
N(u)=P_{1}[(u \cdot \nabla) u] .
\end{array}\right.
$$

We notice that $L: H^{2+\alpha}(T M) \rightarrow H^{\alpha}(T M)$ is a bounded linear operator corresponding to the standard Stokes equation, and $N: H^{2+\alpha}(T M) \rightarrow H^{\alpha}(T M)$ is a $C^{\infty}$ nonlinear compact operator. As in [31], we can prove that there is an open and dense set $\mathcal{F}$ of $H^{\alpha}(T M)$ such that for any $g \in \mathcal{F}$, all corresponding steady states are structurally stable in $H^{2+\alpha}(T M)$, and the mapping $G: H^{2+\alpha}(T M) \rightarrow H^{\alpha}(T M)$ is locally homeomorphic. More precisely, we have

Theorem 6.2. There exists an open and dense set $\mathcal{F}$ of $H^{\alpha}(T M)$ such that for any $g \in \mathcal{F}$,

1. the corresponding steady states of

$$
P_{1}[v \cdot \nabla v]-\triangle v=g
$$

are given by $v_{i} \in B^{2+\alpha}(T M)(i=1, \cdots, I(g))$ for some integer $I(g)$,

2. there exist open neighborhoods $N_{i} \subset X_{0}$ of $v_{i}$, and an open neighborhood $N(f)$ of $g$ in $H^{\alpha}(T M)$ such that for each $i=1, \cdots, I(g)$,

$$
G: N_{i} \rightarrow N(g)
$$

are diffeomorphisms. Here $X_{0} \subset H^{2+\alpha}(T M)$ is the set of all structurally stable Hamiltonian vector fields in $H^{2+\alpha}(T M)$.

The proof is achieved using the infinite dimensional sard theorem and $C^{2+\alpha}$ estimates of steady state solutions of the 2D Navier-Stokes equations. Since is parallel to a similar result we obtained in [31], we omit the details of the proof.

Back to the Taylor field $v_{n m}$, obviously, $P_{1} f$ given in $(6.12)$ is in $H^{\alpha}(T M)-\mathcal{F}$, the complement of an open and dense subset. $P_{1} f$ can be approximated by elements in $\mathcal{F}$, and those corresponding steady state solutions close to the Taylor field $v_{n m}$ are characterized in Theorem 4.1 .

The time dependent case. Now we study the structural time evolution of the solutions of the Navier-Stokes equations with the Taylor field as an initial data. Let

$$
\left\{\begin{array}{l}
u(\cdot ; 0)=v_{n m}, \\
f_{0}=v_{n m} \cdot \nabla v_{n m}-\Delta v_{n m}+\nabla p_{0} \\
f=f_{0}+v+\nabla \phi, \\
v \in H^{r}(T M), \quad \phi \in C^{r+1}(M),
\end{array}\right.
$$

where $v_{n m}$ is the Taylor field. Then the solution $u(x, t)$ of $(6.1-6.3)$ with initial condition $u(\cdot ; 0)=v_{n m}$ can be expressed near $t=0$ as

$$
u(x, t)=v_{n m}+t v+o(t)
$$

Therefore, it is expected that there is an open and dense set $\mathcal{S} \subset H^{r-2}(T M) \oplus G^{r-2}(T M)$ such that for any $f \in \mathcal{S}$ there is a $t_{0}>0$ such that the solution $u(\cdot, t) \in H_{s}^{r}(T M)$ for any $t \in\left(0, t_{0}\right)$. Studies along this direction will be conducted elsewhere. 
Acknowledgments. The authors are grateful to G. Papanicolaou for suggesting us to study the Taylor vortices, and to anonymous referee for pointing out the connections of our results with the measured foliation theory introduced by W. Thurston [52]. The work was supported in part by the Office of Naval Research under Grant NAVY-N00014-96-1-0425, by the National Science Foundation under Grant NSF-DMS-9623071, and by the National Science Foundation of China under Grant 19971062.

\section{REFERENCES}

[1] R. Abraham and J. Marsden, Foundations of Mechanics, Addison-Wesley: Reading, MA (1978).

[2] D.V. Anosov and V. Arnold, Dynamical Systems I, Springer-Verlag, New York, Heidelberg, Berlin (1985).

[3] V. Arnold, Mathematical Methods of Classical Mechanics, Springer-Verlag, New York, Heidelberg, Berlin (1978).

[4] Alain Bensoussan, Jacques-Louis Lions and Papanicolaou George, Asymptotic analysis for periodic structures, Ser. Studies in Mathematics and its Applications. 5; North-Holland Publishing Co., Amsterdam (1978) 700.

[5] D. Chillingworth, Differential topology with a view to applications. Pitman, London, San Francisco, Melbourne. Research Notes in Mathematics, 9 (1976).

[6] A. Chorin, Vorticity and Turbulence, Springer-Verlag (1994).

[7] P. Constantin and C. Foias, The Navier-Stokes Equations, Univ. of Chicago Press, Chicago (1988).

[8] L. Caffarelli and R. Kohn and L. Nirenberg, On the regularity of the solutions of Navier-Stokes Equations. Comm. Pure Appl. Math. 35 (1982) 771-831.

[9] Strebel, Kurt, Quadratic differentials, Springer-Verlag, Berlin (1984) 184.

[10] A. Fathi, F. Laudenbach and V. Poénaru, Travaux de Thurston sur les surfaces. Asterisque 66-67 (1979).

[11] A. Fannjiang and G. Papanicolaou, Convection enhanced diffusion for periodic flows. SIAM J. Appl. Math. 54 (1994) $333-408$.

[12] H. Hopf, Abbildungsklassen n-dimensionaler mannigfaltigkeiten. Math. Annalen 96 (1926) 225-250.

[13] D. Gottlieb, Vector fields and classical theorems of topology. Rendiconti del Seminario Matematico e Fisico, Milano 60 (1990) 193-203.

[14] J. Milnor, Topology from the differentiable viewpoint. University Press of Virginia, based on notes by D.W. Weaver, Charlottseville (1965).

[15] J. Guckenheimer and P.J. Holmes, Nonlinear oscillations, dynamical systems, and bifurcations of vector fields, Springer-Verlag, New York, Heidelberg, Berlin (1983).

[16] J.K. Hale, Ordinary differential equations, Robert E. Krieger Publishing Company, Malabar, Florida (1969).

[17] M.W. Hirsch, Differential topology, Springer-Verlag, New York, Heidelberg, Berlin (1976).

[18] J.L. Lions, Quelques Méthodes de Résolution des Problèmes aux Limites Non Linéaires, Dunod, Paris (1969).

[19] A. Katok and B. Hasselblatt, Introduction to the Modern Theory of Dynamical Systems, Cambridge University Press (1995).

[20] J. Leray, Étude de diverses équations intégrales non linéaires et de quelques problèmes que posent l'hydrodynamique. J. Math. Pures et Appl. XII (1933) 1-82.

[21] J.L. Lions, R. Temam and S. Wang, New formulations of the primitive equations of the atmosphere and applications. Nonlinearity 5 (1992) 237-288.

[22] J.L. Lions, R. Temam and S. Wang, On the Equations of Large-Scale Ocean. Nonlinearity 5 (1992) 1007-1053.

[23] J.L. Lions, R. Temam and S. Wang, Models of the coupled atmosphere and ocean (CAO I). Computational Mechanics Advance, 1 (1993) 3-54.

[24] J.L. Lions, R. Temam and S. Wang, Geostrophic Asymptotics of the Primitive Equations of the Atmosphere. Topological Methods in Nonlinear Analysis 4; note "Special issue dedicated to J. Leray" (1994) 253-287.

[25] J.L. Lions, R. Temam and S. Wang, Mathematical study of the coupled models of atmosphere and ocean (CAO III). J. Math. Pures Appl. 73 (1995) 105-163.

[26] J.L. Lions, R. Temam and S. Wang, A Simple Global Model for the General Circulation of the Atmosphere, "Dedicated to Peter D. Lax and Louis Nirenberg on the occasion of their 70th birthdays". Comm. Pure. Appl. Math. 50 (1997) $707-752$.

[27] P.L. Lions, Mathematical Topics in Fluid Mechanics, Oxford science Publications (1996).

[28] A. Majda, Vorticity and the mathematical theory of incompressible fluid flow. Frontiers of the mathematical sciences: 1985 (New York). Comm. Pure Appl. Math. 39 (1986) S187-S220.

[29] T. Ma and S. Wang, Dynamics of Incompressible Vector Fields. Appl. Math. Lett. 12 (1999) 39-42.

[30] T. Ma and S. Wang, Dynamics of 2-D Incompressible Flows. Proceedings of the International Conferences on Differential Equations and Computation (1999).

[31] T. Ma and S. Wang, The Geometry of the Stream Lines of Steady States of the Navier-Stokes Equations. Contemporary Mathematics, AMS 238 (1999) 193-202.

[32] T. Ma and S. Wang, Block structure and stability of 2-D Incompressible Flows (in preparation, 1999).

[33] T. Ma and S. Wang, Structural classification and stability of divergence-free vector fields. Nonlinearity (revised, 1999).

[34] A. Majda, The interaction of nonlinear analysis and modern applied mathematics. Proc. Internat. Congress Math., Kyoto, 1990, Springer-Verlag, New York, Heidelberg, Berlin (1991) Vol. 1.

[35] N. Markley, The Poincaré-Bendixson theorem for Klein bottle. Trans. AMS 135 (1969). 
[36] L. Markus and R. Meyer, Generic Hamiltonian systems are neither integrable nor ergodic. Memoirs of the American Mathematical Society 144 (1974).

[37] J. Moser, Stable and Random Motions in Dynamical Systems. Ann. Math. Stud. No. 77. Princeton (1973).

[38] J. Palis and W. de Melo, Geometric theory of dynamical systems, Springer-Verlag, New York, Heidelberg, Berlin (1982).

[39] J. Palis and S. Smale, Structural stability theorem. Global Analysis. Proc. Symp. in Pure Math. XIV (1970).

[40] M. Peixoto, Structural stability on two dimensional manifolds. Topology 1 (1962) 101-120.

[41] C. Pugh, The closing lemma. Amer. J. Math. 89 (1967) 956-1009.

[42] Shub, Michael, Stabilité globale des systèmes dynamiques. Société Mathématique de France. Note With an English preface and summary. Astérisque $\mathbf{5 6}$ (1978) iv+211.

[43] C. Robinson, Generic properties of conservative systems, I, II. Amer. J. Math. 92 (1970) 562-603 and 897-906.

[44] C. Robinson, Structure stability of vector fields. Ann. of Math. 99 (1974) 154-175.

[45] C. Robinson, Structure stability of $\mathrm{C}^{1}$ diffeomorphisms. J. Differential Equations 22 (1976) $28-73$.

[46] G. Schwartz, Hodge decomposition-A method for solving boundary value problems. Lecture Notes in Mathematics 1607 Springer-Verlag (1995).

[47] S. Smale, Differential dynamical systems. Bull. AMS 73 (1967) 747-817.

[48] F. Takens, Hamiltonian systems: generic properties of closed orbits and local perturbations. Math. Ann. 188 (1970) $304-312$.

[49] G.I. Taylor, Stability of a viscous liquid contained between two rotating cylinders. Phil. Trans. Roy. Soc. A 223 (1923) $289-343$.

[50] R. Temam, Navier-Stokes Equations, Theory and Numerical Analysis, 3rd edition, North Holland, Amsterdam (1984).

[51] R. Thom, Structural Stability and Morphogenesis, Benjamin-Addison Wesley (1975).

[52] W. Thurston, On the geometry and dynamics of diffeomorphisms of surfaces. Bull. AMS 19 (1988) 417-431.

[53] V. Trofimov, Introduction to Geometry on Manifolds with Symmetry, MIA Kluwer Academic Publishers (1994).

[54] S. Wiggins, Introduction to Applied Nonlinear Dynamical Systems and Chaos, Springer-Verlag, New York, Heidelberg, Berlin (1990).

[55] J.C. Yoccoz, Recent developments in dynamics, in Proc. Internat. Congress Math., Zurich (1994), Birkhauser Verlag, Basel, Boston, Berlin (1994) 246-265 Vol. 1. 